\title{
LE CRIMINEL SAISI PAR LA PSYCHOLOGIE
}

\section{Un entretien AVEC Marc RenNeville ${ }^{1}$}

1. Maîtrẹ̀ de conférences des Universités,

chargé de mission "Histoire" à l'Ecole nationale d'administration pénitentiaire

\author{
Correspondance : \\ Direction des enseignements \\ Ecole nationale d'administration pénitentiaire \\ 440 avenue Michel Serres \\ BP 28 \\ 47916 Agen cedex 9 \\ e-mail : marc.renneville@justice.fr
}

PSN - Comme historien, vous vous intéressez aux rapports entre le crime et la folie. D'où vous est venu cet intérêt?

Marc Renneville (MR) - Mon intérêt sur la question du rapport du crime et de la folie vient d'une recherche sur les sources de la "médecine du crime", c'est-à-dire de l'idée que le crime puisse être une maladie, le criminel un malade, la peine un traitement. Cette idée s'est développée dans les sciences de l'homme (anthropologie, psychiatrie, psychologie...) au $\mathrm{XIX}^{\mathrm{e}}$ siècle. Le rapport du crime et de la folie est une question structurante pour les nouveaux savoirs qui prennent pour objet le crime et, plus encore, le criminel. De Gall à Lombroso, de la question de la monomanie homicide à celle des demi-fous, de l'irresponsabilité pénale à la responsabilité diminuée, on ne cesse de chercher le critère qui permettrait de distinguer nettement la figure du criminel de celle du fou. L'autre source est l'œuvre de Michel Foucault. Quelles que soient les réserves et les critiques qu'on puisse lui adresser, Foucault a produit une riche "boîte à outils" pour (re)penser l'histoire de la psychiatrie, de la prison, de la criminologie. On ne peut reprendre ces questions sans rencontrer les travaux de Foucault.

PSN - De quand peut-on dater l'intérêt de la Justice pour la psychologie des criminels et donc le recours à l'expertise de la médecine mentale?

MR - En un sens, la Justice ne peut se passer d'une psychologie implicite du criminel. Elle suppose en effet un état mental compatible avec l'exercice du droit de punir. Sous l'Ancien Régime, deux catégories de personnes possèdent un statut spécifique du point de vue de la responsabilité civile et pénale: il s'agit des enfants et des fous. Lexistence de commis aux rapports est attestée en France dès le XIII ${ }^{e}$ siècle en France et l'histoire de la médecine légale et de l'expertise psychiatrique retient comme ouvre fondatrice les Questiones medico-legales (1621) de Paolo Zacchias (1584-1659).

Zacchias était appelé à donner son avis d'expert sur les affaires les plus diverses du tribunal papal de la Rote. Il était consulté tant pour des questions purement théologiques, comme l'origine miraculeuse d'une guérison, que pour déterminer si telle affection d'un accusé le dispensait de la tor- ture, si une femme ayant accouchée d'un enfant ressemblant à un animal domestique devait être accusée du crime de bestialité, si tel individu était décédé naturellement ou par empoisonnement etc. Zacchias n'était pas non plus ignorant en matière de nosographie mentale et son art du diagnostic forçait encore le respect des psychiatres de la fin du XIXe siècle (Génil-Perrin notamment), car il admettait des degrés dans l'imputabilité des individus.

Zacchias, pourtant, reste une exception. Sous l'Ancien Régime, la détermination de l'état mental est une question qui échappe généralement au médecin, sauf cas particulièrement litigieux. Dès lors que l'imputabilité pénale est établie, le juge s'intéresse moins à scruter la psychologie du criminel que les circonstances de l'acte car ce sont ces dernières qui vont lui permettre de moduler la peine. $\mathrm{La}$ place que notre système judiciaire accorde à la psychologie du criminel et à la médecine mentale dans la détermination de la sanction est donc assez récente. Elle a été gagnée peu à peu, sur fond de débat entre les "sciences humaines" et le droit, au XIXe siècle, lorsque le problème de la frontière entre la folie et le crime s'est posé avec acuité. 


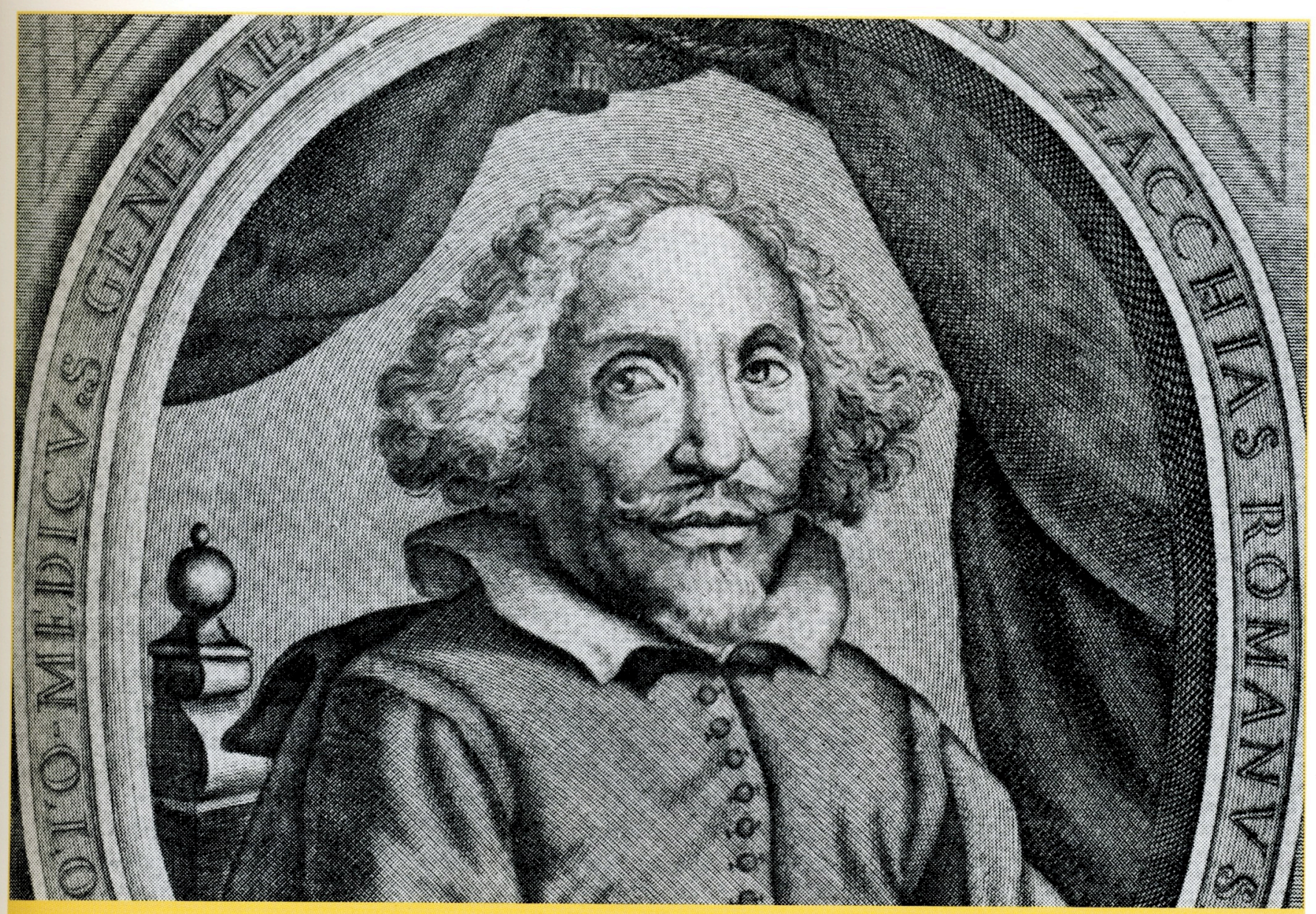

PAOLO ZACCHIA (1584-1659)

Détail d'une gravure par Nicolaus Person, Mayence, 1688

In C. Vallon et G. Genil-Perrin, La psychiatrie médico-légale dans l'oeuvre de Zacchias, Paris, O. Doin et Fils, 1912

Source : Bibliothèque Interuniversitaire de Médecine, Paris, Cote 27323

PSN - Que s'est-il passé à cette période pour que le recours aux "sciences humaines" soit devenu plus aigu, et quelles en ont été les modalités?

MR - Le XIXe siècle débute avec de belles assurances: le code pénal de 1810 réaffirme avec force la distinction du fou et du criminel déjà acquise dans la jurisprudence de l'ancien droit. Rappelons l'article 64 : "Il n'y a ni crime ni délit, lorsque le prévenu était en état de démence au temps de l'action, ou lorsqu'il a été contraint par une force à laquelle il n'a pu résister". Dans le même temps, ce qui deviendra la psychiatrie pose, avec Philippe Pinel, que la folie est une maladie qui peut, dans la plupart des cas, être guérie, car il reste chez les aliénés un fond de raison autorisant la mise en œuvre d'un traitement moral singulier. Ce qui est important ici, c'est que Pinel confère au fou un statut de malade justifiant qu'il soit soustrait à toute incrimination. La part de raison subsistant dans l'aliéné n'autorise pas de sanction pénale, fût-elle diminuée. En ce début de siècle, aliénistes et magistrats ont donc en partage une vision tranchée de la responsabilité : il n'existe aucun degré entre la pleine responsabilité et l'irresponsabilité totale. Ce cadre bien fixé est pourtant remis en cause par le champ médico-légal dans les années 1820 à partir de quelques affaires qui défraient la chronique judiciaire. Toute l'attention se porte sur la personnalité des coupables car les crimes: commis heurtent la morale publique : viol, meurtres d'enfants, actes de cannibalisme. Les mobiles allégués par les accusés semblent bien futiles et inconsistants au regard des forfaits commis. La médecine mentale va alors être convoquée, soit au cours de l'instruction, par le magistrat instructeur, soit lors du jugement, par l'avocat de l'accusé. Certains aliénistes, et le plus connu reste Georget, un élève 
d'Esquirol, vont commenter ces affaires, en expliquant que la justice a affaire à une type très particulier de maladie mentale, qui pousse à commettre des crimes c'est la monomanie homicide. Cette entité clinique est le premier point de contact au XIXe siècle entre la folie et le crime. Un point qui, depuis, n'a cessé de s'élargir. La monomanie homicide est une folie partielle et peut prendre deux formes. Dans la première, le passage à l'acte est causé par une "conviction intime mais délirante", par l' "exaltation" et un "raisonnement fou"; dans la seconde, l'individu ne présente apparemment aucune altération de l'intelligence ni de l'affection, mais il est "entraîné par un instinct aveugle, par quelque chose d'indéfinissable qui le pousse à tuerl". Le délire est alors limité à une discordance entre la sphère instinctive du sujet et sa volonté.

PSN - Dans votre récent ouvrage Crime et folie (Fayard, 2003), vous évoquez certains de ces cas qui ont défrayé la chronique au XIX ${ }^{\mathrm{e}}$ siècle. Pouvez-vous nous en décrire quelques-uns?

MR - Le point commun de ces cas est le caractère abominable des actes et l'apparente normalité de leurs auteurs. Ces affaires interrogent la nature humaine: l'abomination est-elle compatible avec un esprit normal ? La psychiatrisation du passage à l'acte est un moyen de réduire le scandale public par une assignation causale. Ces affaires d' "ogres d'archives" sont bien connues des historiens depuis l'édition du dossier de Pierre Rivière. Dans ces procès, les avocats de la défense ont plaidé la folie de leur client mais l'article 64 n'a pas été appliqué par les magistrats car l'état de démence n'était pas, selon eux, constitué. Je pourrais citer trois exemples:

En 1817, Salomé Guiz est jugée aux Assises de Strasbourg pour avoir assassiné son plus jeune enfant et découpé une cuisse qu'elle a servi dans le souper. On s'est demandé au procès si le crime n'était pas partiellement compréhensible - tout en restant complètement répréhensible - par la disette qui sévissait dans la région, mais l'infanticide possédait encore quelques légumes, des poules et une chèvre. Le docteur François-Emmanuel Fodéré la déclare folle - non sans trouble "pour l'honneur de l'humanité2". Affaire Antoine Léger. Ce jeune vigneron comparait le 23 novembre 1824 devant la Cour d'Assises de Versailles pour avoir tué, violé et mutilé une jeune fille de 12 ans et demi. Léger s'est acharné sur le corps de sa victime. buvant son sang et suçant son cœur. Il n'invoque pas de motifs constants sur la raison de son acte. Il ne connaissait pas sa victime et son acte de cannibalisme ne peut s'expliquer par la faim. A la demande de l'avocat commis d'office, la question de la démence est posée au jury, qui répond négativement. Léger est condamné à mort et exécuté. Sa tête est examinée par le phrénologiste FrançoisJoseph Gall, qui y trouve l'organe du crime.

Louis-Auguste Papavoine, ancien commis de marine, quarante et un ans, comparait aux Assises de Paris les 24,25 et 26 février 1825 pour avoir poignardé deux enfants dans le bois de Vincennes. Cette fois encore, Papavoine ne présente pas d'explication plausible pour ce double assassinat mais son jeune avocat décide d'exposer au jury la théorie de la monomanie homicide. Il sait que la difficulté de l'exercice est de parvenir à concilier dans l'esprit des jurés l'existence de l'aliénation mentale - du délire au moment de l'acte - avec la présence d'esprit manifestée par l'accusé avant et après l'assassinat, mais il ajoute que qualifier pénalement son acte reviendrait à admettre qu'il s'agit d'un crime sans motif. Il poursuit ainsi : "je ne crains pas d'être démenti en disant que ce serait le premier de ce genre depuis qu'il s'en commet ici bas. Un crime sans motif? Êtes-vous bien frappés, Messieurs les Jurés, de tout ce qu'il y a de sens dans ces mots: Un crime sans motif ! et quel crime !". Il rejoint l'argument du docteur Fodéré : "En déclarant que l'accusé n'a point agi volontairement, vous rassurez la société, vous la consolez, vous lui apprenez que ce crime, qui l'avait glacée d'effroi, s'est dépouillé à vos yeux de ce qu'il avait d'horrible, pour retomber dans la classe des accidents ordinaires" 3 ". Lavocat plaide la détention perpétuelle. En vain. Papavoine est condamné à mort et exécuté. Sa tête est récupérée au cimetière par les médecins, qui procéderont à des expériences de galvanisme. Son moulage crânien ira enrichir les collections du cabinet d'anatomie comparée de la Faculté de médecine de Paris.

PSN - Comment le diagnostic de monomanie homicide a-t-il été contesté, qu'en reste-t-il aujourd'hui ?

MR - Les juristes Dupin et Tardif prononcent en 1826 un jugement incisif qui résonnera encore au milieu du siècle dans la mémoire collective des aliénistes : "La monomanie est une ressource moderne; elle serait trop commode, tantôt pour arracher les coupables à la juste sévérité des lois,
1. J.-E.-D. Esquirol, Des maladies mentales..., 1838, 2, p. 791-93.
2. F.-E. Fodéré, Essai médico-légal sur les diverses espèces de folies, Strasbourg, 1832, p. 204.
3. Alphonse Paillet, Plaidoyer pour Auguste Papavoine accusé d'assassinat, Paris, Warée, 1825, p. 47 


\section{Soxfelicatione. \\ des ocganes figures sus cette plandie.}

Penchants.
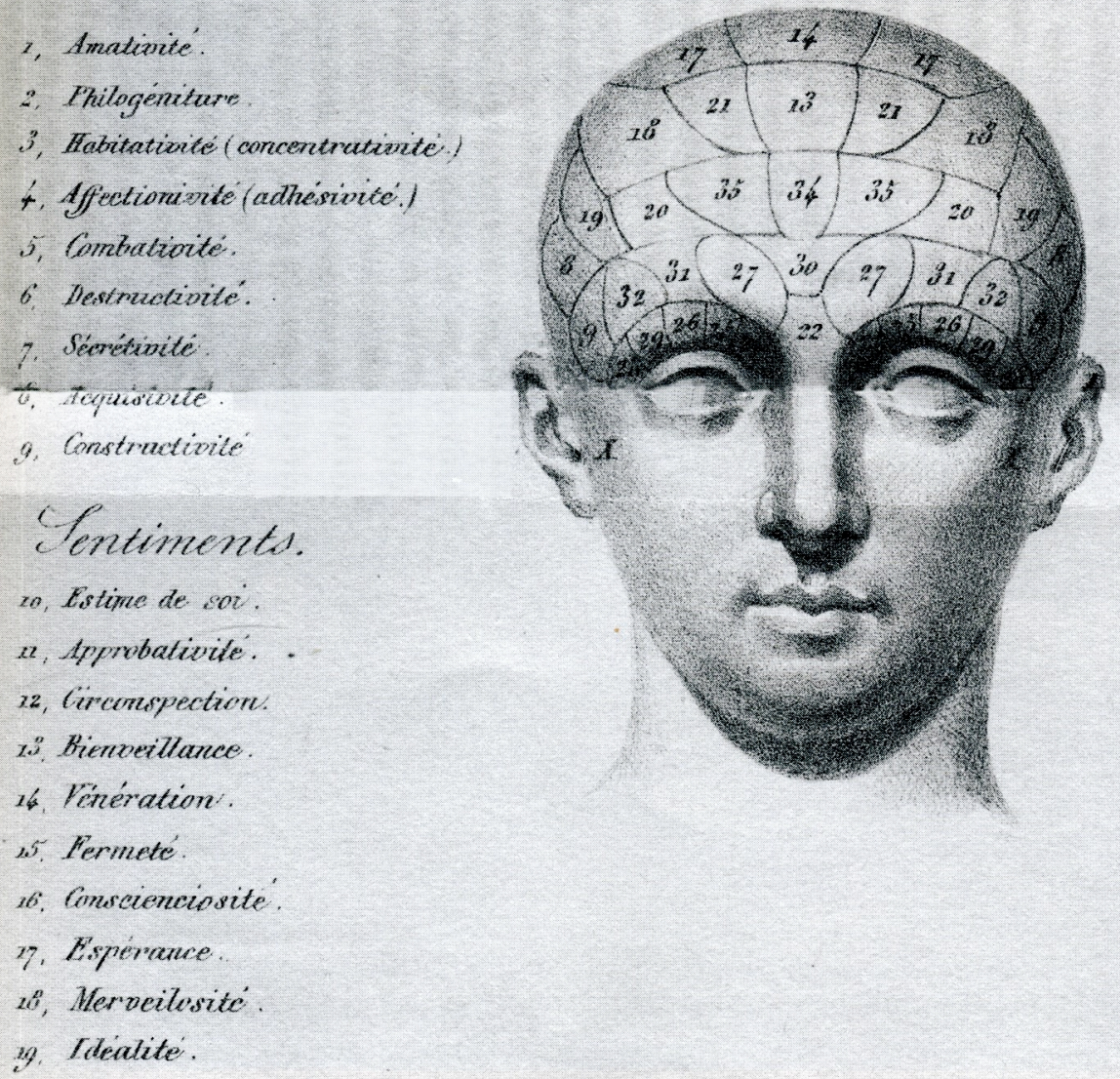

20, Gaibe on asprit de scallice 22. Initation.

traculted feresplives. 22, Individuative

223, Configurution

24. Elendure

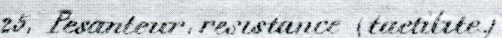

26. Colorits.

27. Lacalité.

26. Cralcid.

39. Ordive

3o, Livenvidite.

.7., Temps.

32, Tots

33, trangoge

raculted iffectiver

34. Comparacison

35. Carzalite
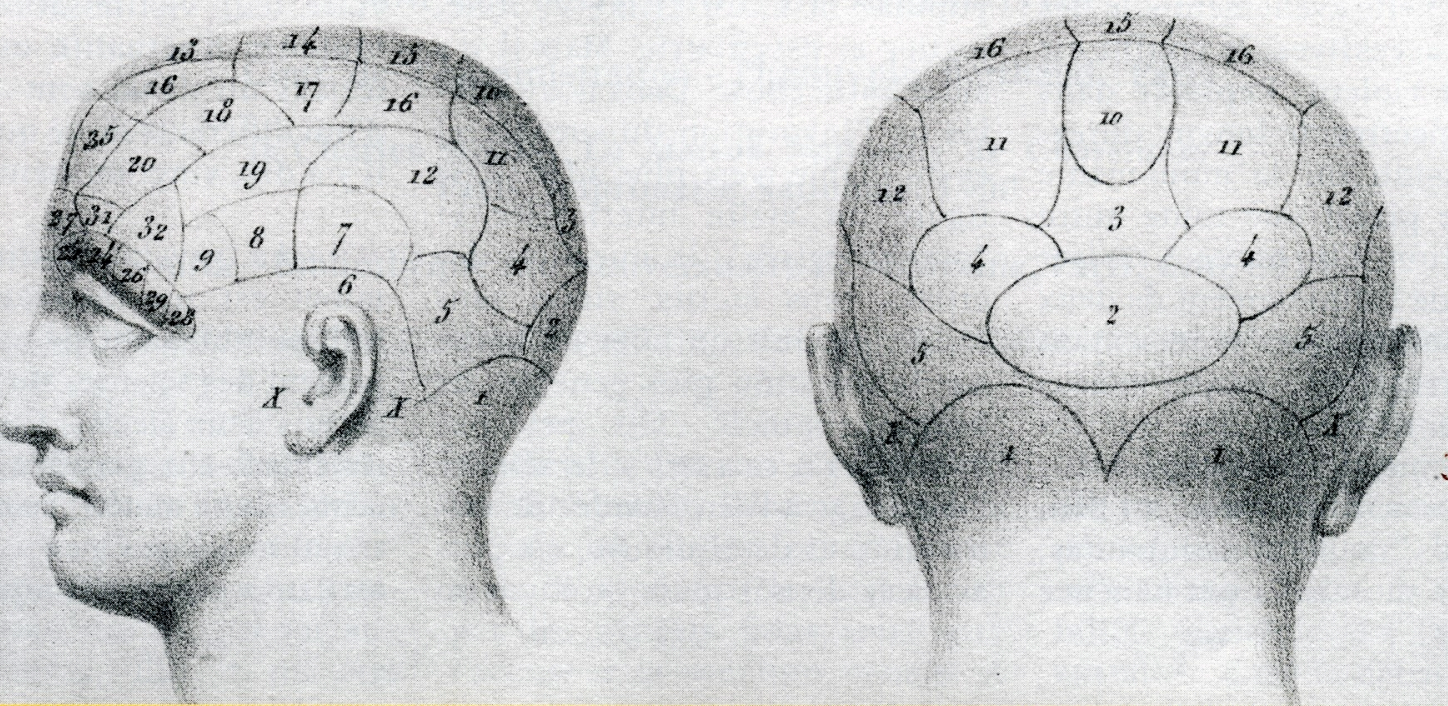

\section{LOCALISATION PHRÉNOLOGIQUE DES FONCTIONS DE L'ESPRIT HUMAIN}

In François-Joseph-Victor Broussais (1772-1838),

Leçons de phrénologie, Bruxelles, Société Encyclographyque des Sciences Médicales, 1839

Source : Bibliothèque Interuniversitaire de Médecine, Paris, Cote 84526 
tantôt pour priver arbitrairement le citoyen de sa liberté. Quand on ne pourrait pas dire : il est coupable, on dirait : il est fou ; et bientôt on verrait Charenton remplacer la Bastille" ". Cette analogie entre la maison royale de Charenton et la Bastille ne désigne pas seulement la crainte de la conquête du pouvoir judiciaire par le corps médical, mais aussi, et peutêtre surtout, celle d'un retour à cet "arbitraire" tant critiqué au XVIIIe siècle. Les opposants à la monomanie homicide estiment qu'elle favorise la confusion de la folie avec le crime, de l'aliénation avec les passions. Ils la jugent infondée en droit puisque la loi n'établit pas de distinction entre la volonté libre et la volonté aliénée. La monomanie homicide semble légitimer une pratique paradoxale puisqu'elle a pour conséquence de soustraire à la répression pénale ceux-là même qui sont poussés au crime par un irrésistible penchant $^{5}$. Et puis, quand bien même le diagnostic pathologique serait assuré, à quoi bon entretenir tant d'égards pour de dangereux incurables? Quelle différence entre peine de mort et détention perpétuelle?

A l'échelle événementielle des affaires successives, le conflit est patent et violent. Replacée dans une perspective de longue durée, la discussion sur la monomanie homicide prend une toute autre signification. Elle incite à réviser le cliché de l'affrontement de deux blocs monolithiques représentant deux mentalités incommensurables, portées par des intérêts professionnels divergents. Les lignes de fractures traversent bien plutôt les deux communautés, comme le montre la permanence du débat en criminologie sur la question des rapports folie/criminalité. Ce débat sur la monomanie homicide marque le premier temps fort dans l'acculturation des normes médicales et judiciaires. Il a mis en lumière les contradictions de la théorie de l'aliénation mentale portée par la génération de Pinel et d'Esquirol. La polémique sur les signes cliniques de la folie criminelle et de la folie du crime a traversé les communautés professionnelles. Au-delà des divergences sur l'art de rendre la justice, elle a permis l'émergence d'un accord sur la nécessité de maintenir l'ordre. Au cœeur d'un débat tout aussi vigoureux à la fin du siècle lorsqu'elle sera théorisée par les positivistes sous le terme de "défense sociale", l'idée de défendre la société est déjà là, omniprésente. Que l'on épouse ou que l'on rejette les positions de Georget sur la justice pénale, on défend l'ordre social et la sécurité publique.

Ce qui reste encore de ce moment, c'est un dossier ouvert sur la nature de la sanction, sur la "pureté" clinique, sur le "sens" de la peine et les bonnes "pratiques", sur l'affinement continu de cette population entre crime et folie, justice et psychiatrie. Mais il y a aussi autre chose. Dans nos sociétés où l'invocation du surnaturel et du diable n'est plus admise, la figure du malin intériorisée et "psychologisée", la question des crimes sans motifs, sinon sans cause, produit un effet de loupe sur la question plus générale de l'agir criminel6. On peut se demander à ce sujet si la science du passage à l'acte, bardée de statistiques et de cliniques, est plus avancée de nos jours qu'il y a un siècle. Dans son dernier roman, Emmanuel Loi nous offre un essai introspectif à la recherche de la logique d'action de Claude Buffet, auteur d'une sanglante prise d'otages lors d'une tentative d'évasion de la prison de Clairvaux, en 1971, et avant-dernier exécuté en France (1972). Au terme de son enquête, le questionnement reste intact : "Qu'est-ce qui fait d'un homme un monstre, comment devient-on un assassin? Par prédestination, goût de l'outrance, exécration de soi chauffée à blanc, proche de l'idôlatrie en fait, absence forcenée de l'autre? Je ne sais plus après de longs mois de coexistence dans ce cloaque ${ }^{7}$." Je crois que le romancier met ici au jour le refoulé de la criminologie. En avouant au lecteur sa volonté de savoir inassouvie, il nous rappelle que la quête médicale et judiciaire de "l'homme criminel" a de beaux jours devant elle.

PSN - Dans l'avant-propos de votre ouvrage, vous soulignez que l'opposition entre les savoirs et les pratiques juridiques d'un côté, et psychiatriques de l'autre, ne sont ni nouvelles, ni figées. La psychiatrisation des auteurs de crimes, voire leur victimisation, est-elle toujours d'actualité ou bien les termes du débat sont-ils aujourd'hui différents de ce qu'ils étaient il y a dix, vingt ou trente ans?

MR - La "psychiatrisation" du criminel est un mouvement lent, indissolublement lié au développement des sciences du psychisme depuis deux siècles. Confinée initialement aux grandes affaires de crimes sans motifs, cette psychiatrisation a gagné peu à peu toutes les catégories de délinquances, dès
4. Dupin et Tardif, "Consultation. Sur l'exposé de M. Martial d'Arzac, détenu à la Force comme prévenu d'irrévérence envers une princesse, et subsidiairement accusé de monomanie", Gazette des tribunaux, 2 avril 1826, n 134, p. 1-2.
5. Collard de Martigny, "Examen médico-légal. De l'opinion émise par divers médecins sur la monomanie homicide", Nouvelle bibliothèque médicale, 1828, vol. 3, p. 204.

6. R. Muchembled, Une histoire du diable. XIII ${ }_{-}$
XXe siècle, Paris, Seuil, 2000.

7. E Loi, Peine capitale, Paris, Flammarion, 2003, p. 243. 


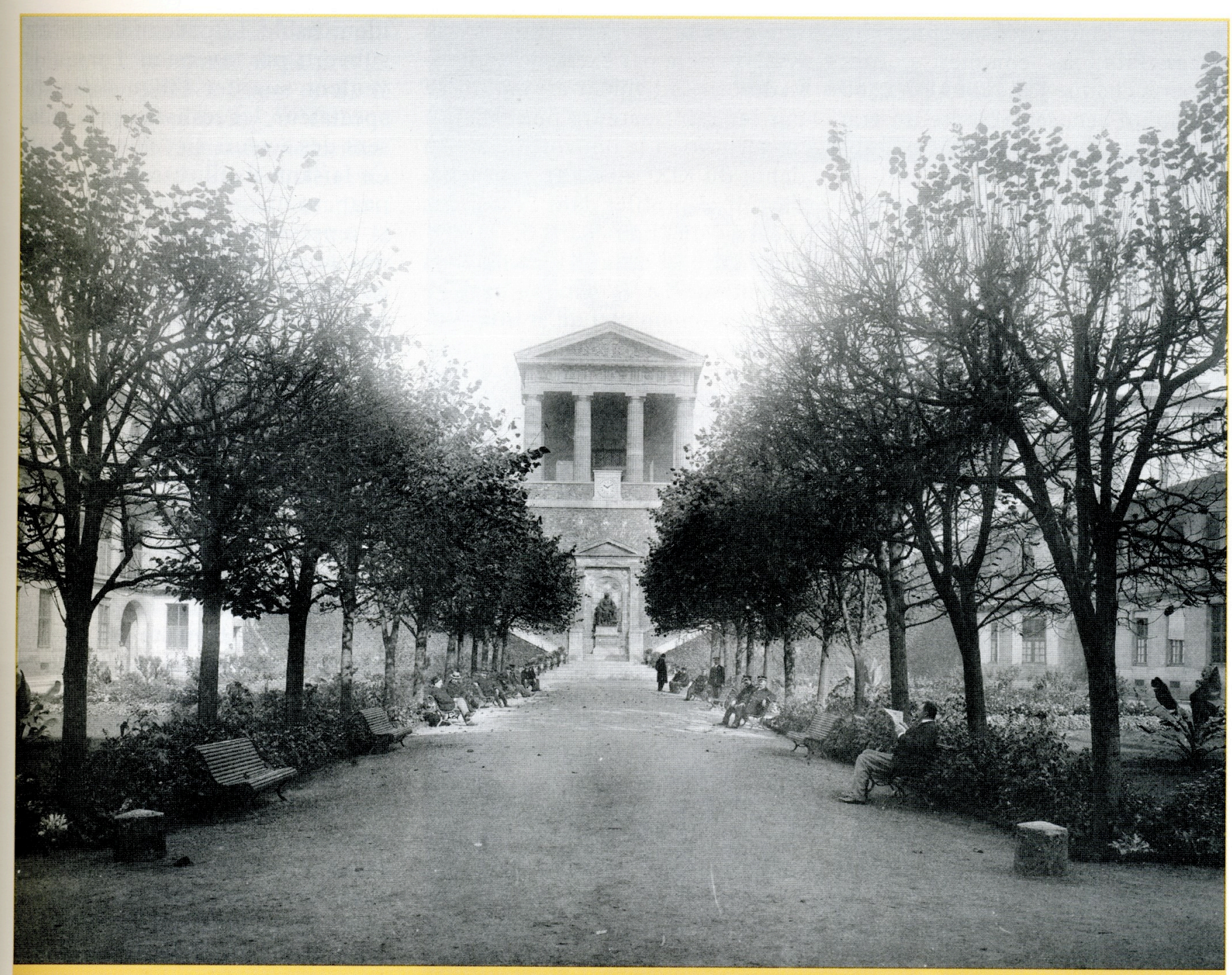

MAISON NATIONALE DE CHARENTON

Cour de l'Administration - Phototypie Berthaud, Paris

In Charles Strauss, La Maison Nationale de Charenton, Paris, Imprimerie Nationale, 1900

Source : Bibliothèque Interuniversitaire de Médecine, Paris, Cote 8914

lors que le recours au simple raisonnement d'intérêt ou de plaisir ne permet pas d'expliquer le passage à l'acte. Cette psychiatrisation a connu des usages sociaux très différents. Elle a pu être mobilisée ici pour sauver la tête d'un accusé, elle a pu être utilisée ailleurs pour dénier toute dimension politique à une opposition au régime en place. Derrière cette diversité, on peut relever une évolution notoire. De Pinel à Morel, la psychiatrisation sert généralement les intérêts de l'accusé, dans la mesure où elle est généralement mise en avant par les experts ou par les avocats pour soustraire l'accusé à la sanction pénale. A partir du milieu du XIXe siècle, certains médecins émettent des diagnostics conciliant, pour un même sujet, anomalies psychiques et responsabilité. Le concept même de "responsabilité" fait l'objet de nombreuses réflexions. A côté de la vieille notion de "libre arbitre", on voit théoriser la "responsabilité diminuée", la "responsabilité sans faute", la "responsabilité sociale". La peine est entraînée dans le débat : s'agit-il de punir l'acte ou de corriger l'individu ? Et dans ce dernier cas, peut-on le redresser, l'amender ou vaut-il mieux l'exclure, le neutraliser, l'éliminer, empêcher sa reproduction? En 1905, le garde des Sceaux semble donner raison 
aux psychiatres en incitant les magistrats à commettre des experts chaque fois qu'un prévenu ou qu'un accusé présente un état mental douteux. Et en cas de rapport constatant l'anomalie, celle-ci doit jouer en faveur d'une diminution relative de la peine. L'esprit et la lettre de cette circulaire marquent bien la distance parcourue depuis lors.

Aujourd'hui, les termes du débat sont bien différents: dans le champ théorique, la psychanalyse a ouvert un horizon neuf d'interprétation; en pratique les experts sont plus réticents de nos jours à reconnaître une irresponsabilité pénale chez les auteurs de crimes. En pratique toujours, le constat d'anomalie psychique peut même jouer dans le sens d'une aggravation du quantum de peine. Il faut ici se mettre à la place du jury qui entend déclarer qu'un accusé est dangereux, qu'il présente des anomalies psychiques et qu'un traitement ne peut garantir une libération sans récidive. L'enfermement de longue durée lui apparaît alors comme la seule solution permettant un minimum de prise de risque pour la société. Peu importe au fond qu'il s'agisse d'enfermer ou de soigner, de prison psychiatrisée ou d'hôpital pénitentiarisé. La victimisation d'un auteur de crime, au fond assez rare, pèse bien trop peu pour compenser ce phénomène.

PSN - Comment la littérature et le cinéma se sont-ils fait l'écho de ces différentes façons de considérer la psychologie du criminel?

MR - Des canards du XVIIe au polar contemporain, du cinéma muet aux films ayant pour sujet des tueurs en série, le crime et le criminel sont des thèmes de prédilection pour la littérature comme pour le cinéma. Lappropriation des thématiques, le traitement même du sujet varie évidemment en fonction du temps et des auteurs. On peut tout au plus relever quelques figures marquantes, quelques temps forts. A la fin du XVIIIe siècle par exemple, la figure du brigand voleur est mobilisée par les réformateurs pour mettre en accusation le pouvoir royal. Au début du XIX ${ }^{e}$ siècle en revanche, on voit se profiler dans l'essor des théâtres du "boulevard du crime" une figure de criminel coupable et repentant. Le roman naturaliste est un moment clef de la littérature pour la psychologie du criminel. Il s'inspire en effet des meilleurs scientifiques de l'époque (Despine, Morel, Lombroso...).

Les savants eux-mêmes prennent certains de ces portraits comme des "documents humains" et s'essaient à la critique littéraire. Le roman feuilleton et la detective novel ne leur conviennent guère et leur préférence va à Zola, Dostoïevski et Ibsen. L'essor du cinéma de fiction au début du $\mathrm{XX}^{\mathrm{e}}$ siècle marque une forte interpénétration de la littérature et du cinéma. La psychologie du criminel est alors très figurative, démonstrative, extériorisée par les attitudes, la physionomie et le jeu de l'acteur. Le théâtre est ici un relais, notamment sur la scène, impasse Chaptal à Paris, du "Grand Guignol", où se joue de courtes pièces sanglantes en un acte sur le thème de la folie meurtrière. Certaines de ces pièces sont d'ailleurs adaptées au cinéma. L'homme mystérieux, fruit de la collaboration d'André de Lorde et Alfred Binet, est réalisé en un moyen métrage par Maurice Tourneur (Obsessions, 1934), avec Charles Vanel, Yonel et Louise Lagrange. Trois ans plus tôt, Fritz Lang avait produit un chef d'cuvre du genre: M. le Maudit, à partir de données tirées de faits divers bien réels.

Ce qui est frappant dans le cinéma parlant, c'est une relative émancipation du stéréotype physionomique qui prévalait dans la science du XIXe. On ne présente généralement pas la folie meurtrière sous la forme d'une déraison complète ou sous le masque d'une physionomie immédiatement identifiable. Le code indiciaire est subverti par inversion. Lorsqu'ils veulent susciter l'inquiétude du spectateur, les réalisateurs mobilisent des acteurs en contre-emploi. Ce faisant, ils illustrent et accompagnent une mutation décisive de la représentation du monstre. De nos jours, le "monstre" est celui qui contredit le rapport traditionnel du moral et du physique. La laideur morale ne correspond pas à la laideur physique. C'est là un des thèmes préférés d'Alfred Hitchcock et on le retrouve encore mis en scène dans le très récent film coréen "Memories of murder" de Bong Joon-Ho (2003). En fait, plus l'individu est proche du commun sur le plan physique, plus il est "monstré" sur le plan psychique. Il y a, bien sûr des acteurs qui se sont spécialisés dans des rôles de "sale gueule". L'exemple le plus frappant n'est pas ici le plus connu. Il s'agit de Rondo Hatton (1894-1946), qui a eu une petite gloire à Hollywood dans quelques rôles de brutes "grâce" à une acromégalie faciale. A bien y regarder toutefois, c'est surtout dans le genre "gore" que l'on vérifie l'adéquation du physique et du moral. Les grands films de criminels brossent des portraits beaucoup plus subtils et plus angoissants parce qu'ils jouent sur un rapport de proximité avec le spectateur, d'identification, voire de fascination - et non d'exclusion.

PSN - A-t-on une idée de l'influence mimétique, sur les criminels, ou cathartique, sur le public, de ces représentations littéraires et cinématographiques?

MR - La question est aussi ancienne que la littérature criminelle elle-même. Elle est très présente dans les sciences du crime à travers l'idée de "contagion" du meurtre. Au XIXe siècle, phrénologistes, psychiatres et psychologues s'accordent à penser qu'une "contagion" des comportements est possible. En 1875, Paul Moreau de Tours publie une étude 
(La Contagion du suicide) en soulignant le rôle néfaste de la presse. Quelques années plus tard, le magistrat criminologue Gabriel Tarde fait de l'imitation le principal facteur de la vie sociale et du crime. Enfin, le docteur Paul Aubry consacre un ouvrage à la contagion du meurtre, en 18948 .

Il est vrai que cette fin de siècle est marquée par une psychologie physiologique faisant la part belle aux phénomènes de suggestion et à l'hypnose. C'est aussi l'âge d'or de la presse de fait divers, saturée par l'imagerie du crime et du criminel. Kiosques et boutiques à journaux affichent en bonne place la gravure représentant le crime du jour à la Une des journaux populaires (Le Petit Journal, L'Intransigeant...) et les romans mettent en récit des crimes et des meurtres en se penchant sur la psychologie du criminel, sa personnalité et ses mobiles. Les savants de l'époque s'accordent toutefois pour penser que le processus de la contagion n'est pas mécanique, de la représentation au passage à l'acte. Paul Aubry distingue ainsi quatre vecteurs possibles de contagion (la famille, la vie en commun des prisonniers, le spectacle des exécutions et la presse) en soulignant que celle-ci ne peut atteindre qu'un individu dont l'organisme est "prédisposé". Ce qui est certain, c'est que le phénomène est bien repéré par le recueil de cas dans lesquels les individus invoquent telle ou telle affaire, telle ou telle lecture pour expliquer leur passage à l'acte. Il en va de même avec le cinéma, dont le pouvoir de persuasion est peut-être plus fort encore. En tout cas, il est très vite dénoncé pour ses effets délétères, notamment sur la jeunesse. La liberté de l'artiste est-elle totale? Faut-il censurer ? Le débat resurgit à chaque film ultra-violent. Stanley Kubrick a connu cette

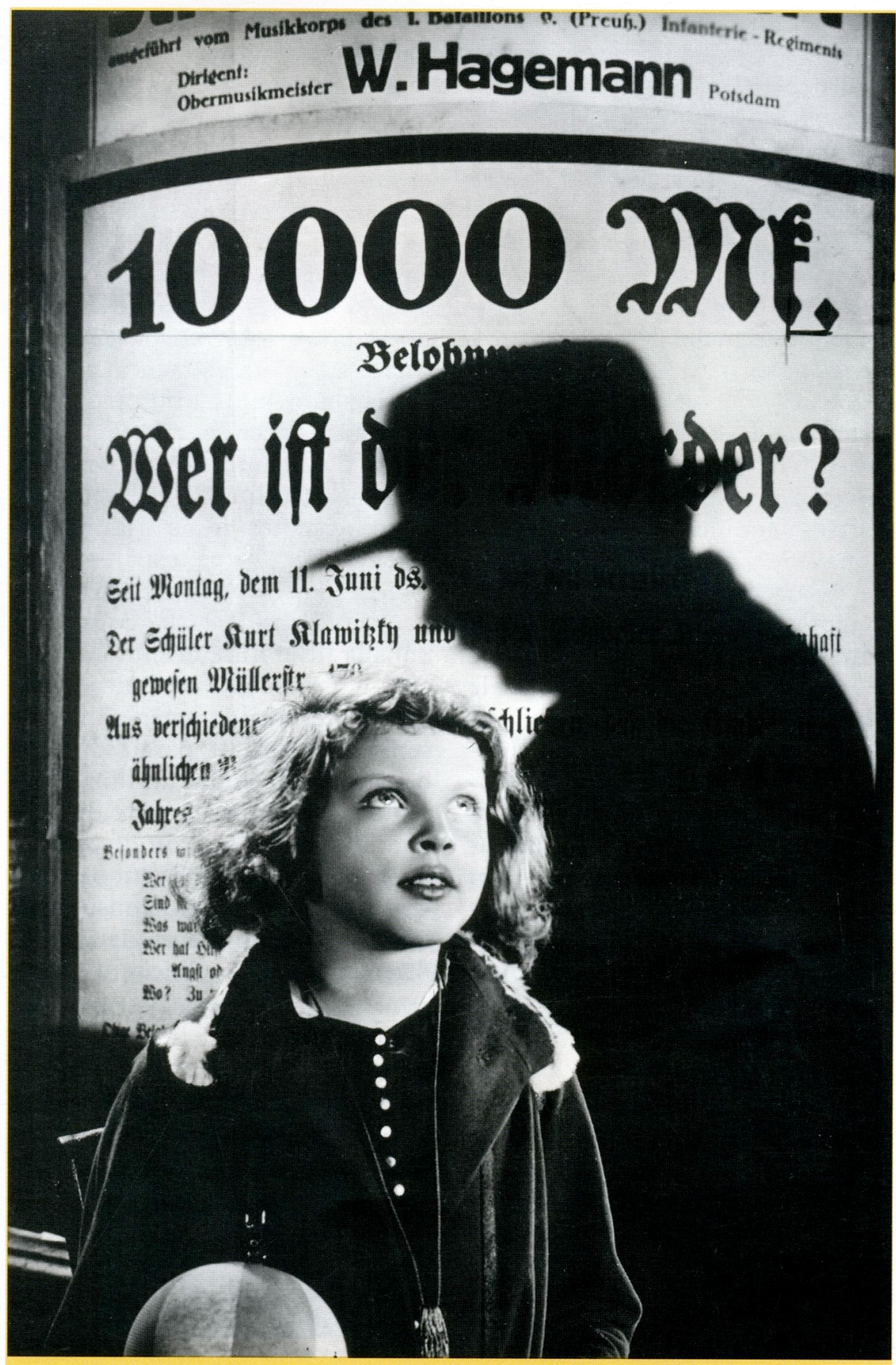

LE MEURTRIER CHOISIT SA VICTIME

Elsie Beckmann, interprétée par Inge Landgut Hans Beckert, alias M, interprété par Peter Lorre In Fritz Lang, M le Maudit,

Seymour Nebenzahl, Nero-Film AG, Berlin, 1931

Source: Iconothèque de la Bibliothèque du Film, Paris Copyright Atlantic-Film AG 
mésaventure avec Orange mécanique (1971), dont la première partie reste, encore de nos jours, quasiment insoutenable. Il a d'ailleurs décidé d'arrêter sa distribution lorsque des bandes de jeunes ont commencé à sévir en endossant les costumes et les comportements de la bande d'Alex. Lès films de Wes Craven ont également déclenché des polémiques. Après la sortie de La Colline a des yeux (1977), le réalisateur s'est défendu de faire l'apologie du crime. On peut se demander toutefois si la responsabilité du créateur est annulée par ces dénégations? Par ailleurs, est-il nécessaire de montrer l'acte pour le représenter? De grands réalisateurs ont opté pour l'ellipse. C'est le cas de Fritz Lang, dans M. le Maudit (1931).

La question ici posée au cinéma, à chaque sortie de film violent, est en réalité la même que celle que l'on posait à la littérature au XIXe siècle: ne risque-t-on pas de donner des idées aux personnes "prédisposées"? Vernon Zimmerman donne une réponse par film interposé en présentant, dans Fondu au noir (1980), un jeune homme cinémaniaque qui passe à l'acte en reproduisant à chaque fois le comportement du criminel de ses films-cultes. Quant à Wes Craven, il répond également sur le ton de la parodie dans Scream 3 (2000). Le cinéaste peut d'ailleurs estimer que si la personne est "prédisposée", son film n'est qu'un élément nécessaire mais non suffisant du passage à l'acte. De récentes affaires continuent d'alimenter cette idée d'une influence "mimétique", notamment avec les films sataniques, Scream etc. Qu'en est-il exactement?

En l'absence de consensus dans la littérature criminologique contemporaine, on ne peut qu'avancer quelques généralités de sens commun. Il paraît difficile d'abord de défendre un film ultraviolent et amoral au nom du seul effet de "catharsis", du divertissement ou de l'effet esthétique alors que notre société de consommation postule la manipulation du désir à travers la publicité. Il paraît tout aussi difficile, a contrario, de renvoyer la violence de notre société ou de certains comportements aux seules influences du "poids des mots" et du "choc des photos". Pour paraphraser le criminologue Alexandre Lacassagne, contemporain des frères Lumière, nos sociétés ont la littérature et le cinéma qu'elles méritent. Enfin, et c'est peut-être ici le seul apport réellement nouveau des sciences humaines, l'effet d'une représentation dépend de l'émetteur et du récepteur, au-delà même des intentions conscientes de l'un et de l'autre. Les conditions même de réception sont à prendre en compte (public/privé, individu isolé ou en groupe...), tout comme la capacité d'analyse critique et de mise à distance de l'individu. Sans nier donc que certaines images très fréquentes puissent avoir une influence sur nos opinions, voire nos comportements, il est très probable, même si ce n'est guère rassurant, qu'une même représentation puisse être "catharsis" chez l'un, "mimésis" chez l'autre, à effet nul chez un troisième.

\section{Directeur de PUbLicATION \\ Luigi Grosso \\ e-mail : luigros@aol.com}

\section{RÉDACTEURS EN CHEF}

Jean-Michel Azorin (Marseille)

Bernard Granger (Paris)

Luigi Grosso (Paris)

\section{COMITÉ dE RÉdACTION}

David Cohen (Paris)

Nicolas Dantchev (Paris)

Bruno Falissard (Paris)

Jean Naudin (Marseille)

Bernard Pachoud (Paris)

Dominique Pringuey (Nice)

Florence Thibaut (Rouen)

Antoine Triller (Paris)

\section{Secrétariat de rédaction}

Professeur B. Granger

Service de Psychiatrie d'adultes Hôpital Necker

149, rue de Sèvres - 75015 Paris e-mail : granger@necker.fr

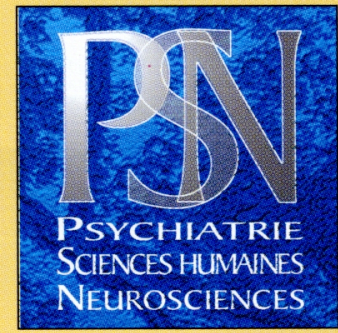

PSN Editions,

SARL au capital de $10500 €$. RCS Paris B en cours. 198, rue de la Convention 75015 Paris.

Tél. : 0156566565

Fax : 0156566566

Périodicité : bimestriel

ISSN : 1639-8319

Commission Paritaire : 0505 T83086

Dépôt légal : ler trimestre 2003

Référencé dans les bases de données PASCAL et FRANCIS

\section{ABOnNEMENTS}

Abonnement 1 an, 6 numéros : médecins, $115 €$ - étudiants, $65 €$ institutionnels, $170 €$

Etranger : ajouter $12 €$ à ces tarifs Prix au numéro : $25 €$

Les demandes d'abonnements sont à adresser, accompagnées de votre règlement par chèque à l'ordre de PSN, 198, rue de la Convention, 75015 Paris.

\section{Fabrication}

PAO : Silicate (Tél. 06614506 75)

Imprimeries de Champagne (Langres)

\section{Régie - Publicité}

Cordée, Paul Aim

198 , rue de la Convention

75015 Paris.

Tél. 0156566565

Fax : 0156566566

ILLUSTRATION DE COUVERTURE Photo (c) Jean-Pierre Bourgeois, Paris 